\title{
Upregulation of sphingosine kinase 1 is associated with recurrence and poor prognosis in papillary thyroid carcinoma
}

\author{
JIE LI, BO ZHANG, YANG BAI, YONGHONG LIU, BUYONG ZHANG and JIAN JIN
}

\author{
The Fourth Department of Thyroid and Breast Surgery, Cangzhou Central Hospital, Cangzhou, Hebei 061000, P.R. China
}

Received November 16, 2018; Accepted July 26, 2019

DOI: $10.3892 / 01.2019 .10910$

\begin{abstract}
Sphingosine kinase 1 (SPHK1), an ATP-dependent protein, has previously been demonstrated to be upregulated in several types of human cancer and to play an important role in tumor development and progression. However, the role of SPHK1 in predicting long-term prognosis in patients with papillary thyroid carcinoma (PTC) remains unclear. The purpose of the present study was to assess the significance of SPHK1 expression and its associations with clinicopathological characteristics and prognostic outcome in patients with PTC. Immunohistochemistry staining was retrospectively performed to investigate the expression levels of SPHK1 in 92 PTC tumors. Statistical analyses revealed that high levels of SPHK1 expression were associated with tumor size, lymph node metastasis and the Tumor-Node-Metastasis stage. The disease-free survival (DFS) time of patients that exhibited high levels of SPHK1 expression was shorter, whereas patients with lower levels of SPHK1 expression survived longer. Furthermore, multivariate analysis suggested that upregulated SPHK1 was an independent prognostic factor for predicting DFS of patients with PTC. The results of the Cell Counting Kit- 8 and invasion assays demonstrated that SPHK1 overexpression significantly enhanced the proliferation and invasion of a PTC cell line, consistent with clinical findings. The results from the present study provide evidence that elevated expression levels of SPHK1 may be involved in the development and progression of PTC, indicating that this protein may act as a potential prognostic marker for patients with this disease.
\end{abstract}

\section{Introduction}

Thyroid cancer is the most common malignancy of the endocrine system and accounted for $\sim 1 \%$ of all human malignancies in 2006 worldwide (1). In numerous countries, the incidence of

Correspondence to: Professor Jie Li, The Fourth Department of Thyroid and Breast Surgery, Cangzhou Central Hospital, 16 Xinhua West Road, Yunhe, Cangzhou, Hebei 061000, P.R. China

E-mail: lijiepw@126.com

Key words: sphingosine kinase 1, papillary thyroid carcinoma, prognosis, survival thyroid cancer has been increasing faster than that of any other malignancy (2). According to a 2015 report by the American Cancer Society, the probability of being diagnosed with thyroid cancer has tripled over the last three decades, making it the most increasingly prevalent cancer in the USA (3). Thyroid cancer consists of the following four histological types: Papillary, follicular, medullary and anaplastic thyroid carcinoma. Different surgical techniques according to the tumor stage and carcinoma type are the primary modes of therapy for thyroid cancer. Papillary thyroid carcinoma (PTC) is the most common pathological type of this cancer, accounting for $\sim 80 \%$ of all cases between 1980 and 2005 in the USA (4). Although thyroidectomy combined with radioactive iodine treatment and chemotherapy has achieved good clinical outcomes, recurrence and metastasis are the main causes of mortality in patients with thyroid cancer $(5,6)$. It is common for PTC to metastasize to the lymph nodes (LNs) of the neck, occurring in up to $50 \%$ of cases $(7,8)$. Therefore, identifying protein markers that are associated with the recurrence, metastasis and prognosis of thyroid carcinoma is particularly important for clinical practice. Such newly discovered markers may also promote the continuous development of efficient treatments for thyroid carcinoma.

Sphingosine kinase (SPHK), an ATP-dependent protein, is expressed in various organisms such as mammals, insects, invertebrates, yeast strains and plants. In mammals, SPHK has been identified to consist of two isozymes, SPHK1 and SPHK2. SPHK1 catalyzes the phosphorylation of sphingosine to produce sphingosine-1-phosphate (S1P), which is a potent lipid mediator involved in a variety of physiological processes, including angiogenesis, cell proliferation, apoptosis, motility and migration (9). Ceramides and sphingosine, the metabolic precursors of S1P, are known to possess regulatory functions in cell apoptosis (10). Thus, the balance between these precursors and S1P within the cell has been suggested to function as a switch that drives the decision between cell proliferation and death (11). However, the key regulator of this switch is SPHK1, which converts sphingosine into prosurvival S1P. Previous studies have demonstrated that the activity of SPHK1 is associated with anti-phagocytosis, cell transformation, proliferation and the survival of tumor cells $(12,13)$. In addition, SPHK1 mRNA is overexpressed in a number of types of solid tumor, including those of the stomach, rectum, kidney, breast, colon, ovary, uterus and lung (14). Other studies have demonstrated that high expression levels of SPHK1 in breast cancer and 
glioblastoma are associated with poor patient prognosis, suggesting that SPHK1 functions as a promoter of cancer in human tumors $(15,16)$. Furthermore, it has been reported that high levels of SPHK1 expression were associated with clinical stages, locoregional recurrence and distant metastasis of nasopharyngeal carcinoma (17). Therefore, SPHK1 may serve distinct roles in different tumor types.

However, to the best of our knowledge, few comprehensive studies exist that have investigated the expression and significance of SPHK1 with respect to the long-term prognosis of patients with thyroid carcinoma. To date, whether SPHK1 is involved in the recurrence of thyroid carcinoma remains unknown. Therefore, the aim of the present study was to investigate the expression of SPHK1 in human PTC tissue samples using immunohistochemistry (IHC), and its association with clinical characteristics and pathological parameters. In addition, the effect of SPHK1 positivity on the recurrence and prognosis of patients with PTC was investigated, assessing its effectiveness as a potential novel prognostic molecular marker for the management of PTC in the clinical setting.

\section{Materials and methods}

Tissue specimens. The protocol and tissue specimen collection from human participants for the present study was approved by the Medical Institutional and Clinical Research Ethics Committee of Cangzhou Central Hospital (Cangzhou, China). Formalin-fixed paraffin-embedded tissue microarrays consisting of 92 samples of PTC were used in this study. The tissue specimens were collected during thyroidectomies from Cangzhou Central Hospital, and the tissue microarrays with these specimens were produced by the Alenabio and Shrbio Biological Technology companies. The patients included in the study met the following criteria: i) Histopathologic diagnosis of PTC; ii) complete clinical data; and iii) patients underwent a total or near-total thyroidectomy for PTC. Written informed consent was previously obtained from the 92 patients diagnosed with PTC who received surgical treatment. Patients who had received radiotherapy or neoadjuvant therapy prior to the surgery were excluded from the study. All patients were followed up, and their clinicopathological parameters and survival times were obtained. The follow-up was conducted primarily via outpatient reviews, telephone, mail and home visit for at least 120 months.

The PTC tumors were staged based on the current Tumor-Node-Metastasis (TNM) classification system (18), and the patients underwent post-operative radioactive iodine ablation, according to the guidelines of the American Thyroid Association (19). Clinical data, including sex, age, tumor size, thyroid capsular invasion (TCI), extrathyroidal extension (ETE), histological type, LN status, distant metastasis, vascular invasion and recurrence state, were retrieved for all patients, as were complete follow-up details.

IHC. PTC tissue microarray slides were deparaffinized in xylene and rehydrated with 70, 80, 90 and 100\% graded ethanol solutions, and then, the endogenous peroxidase activity was inhibited by a $0.3 \%$ hydrogen peroxide solution. The sections were immersed in sodium citrate buffer $(10 \mathrm{mM}$ sodium citrate; $0.05 \%$ Tween-20; $\mathrm{pH} 6.0$ ) at $105^{\circ} \mathrm{C}$ for the antigen retrieval. The sections were then incubated with $5 \%$ goat serum (Solarbio Biotechnology, Inc.) to block the non-specific background peroxidase activity at room temperature for $10 \mathrm{~min}$. Subsequently, the slides were incubated with a rabbit polyclonal anti-human SPHK1 primary antibody (1:100; cat. no. ab71700; Abcam) at $4^{\circ} \mathrm{C}$ overnight. The sections were then incubated with goat anti-rabbit secondary antibody (1:1,000; cat. no. ab7090; Abcam) at $37^{\circ} \mathrm{C}$ for $15 \mathrm{~min}$, and the results were visualized using a diaminobenzidine tetrahydrochloride detection kit (Santa Cruz Biotechnology, Inc.) according to the manufacturer's protocol. Finally, the sections were rinsed for $5 \mathrm{~min}$ in PBS, counterstained with $2.5 \%$ hematoxylin for $5 \mathrm{~min}$ at room temperature and rinsed again with tap water. For negative controls, the antibody was replaced by PBS. Images were captured with an Olympus BX42 light microscope (Olympus Corporation; magnification, $\mathrm{x} 40$ ).

Evaluation of staining. IHC was performed as aforementioned (20). A total of five high-power fields of view of the tumor sections were randomly selected, and 500 cells in each field were counted to determine the labeling index, which represents the estimated percentage of positive cells relative to the total cells. Sections with $<5 \%$ labeled cells were scored as 0 ; sections with 5-30\% labeled cells were scored as 1; 31-70\% labeled cells were scored as 2 ; and sections with $\geq 71 \%$ labeled cells were scored as 3 . Staining intensity was evaluated similarly, with a score of 0 for negative staining; 1 for weak positive; 2 for moderate positive; and 3 for strong positive staining. The scores of the percentage and intensity were added, and final scores of 0-1 indicated negative expression (-), 2-3 indicated weak expression (+), 4-5 indicated moderate expression (++) and 6 indicated strong expression $(+++)$. The sections were evaluated by two pathologists from Cangzhou Central Hospital blinded to the study. For the statistical analysis, staining was classified into high-(score, 4-6) and low-(score, 0-3) expression groups.

Cell culture and transfection. The human PTC TPC-1 cell line was purchased from the Cell Bank of the Chinese Academy of Science. The TPC-1 cells were cultured in Dulbecco's modified Eagle's medium (DMEM; Hyclone; GE Healthcare Life Sciences) supplemented with $10 \%$ fetal bovine serum (FBS) (Gibco; Thermo Fisher Scientific, Inc.), and were maintained in an incubator at $37^{\circ} \mathrm{C}$ with a humidified atmosphere containing $5 \% \mathrm{CO}_{2}$.

For overexpression of the SPHK1 gene, pcDNA3.1 vector was purchased from Thermo Fisher Scientific (cat. no. V79020) and pcDNA3.1-SphK1 was purchased from Shanghai GenePharma Co., Ltd. The pcDNA3.1-SPHK1 plasmid was constructed as previously described (21). The empty vector pcDNA3.1 plasmid was transfected into the cells as a negative control. The PTC TPC-1 cells were seeded in 6 -well plates at density of $5 \times 10^{4}$ cells per well. The cells were transfected with the pcDNA3.1-SPHK1 plasmid once they had reached $\sim 80 \%$ confluence. To transfect the cells, $2 \mu \mathrm{g}$ plasmid and $6 \mu$ l Lipofectamine ${ }^{\circledR} 3000$ solution (Invitrogen; Thermo Fisher Scientific, Inc.) were briefly mixed and incubated with the cells for $5 \mathrm{~min}$ at room temperature. The overexpression experiment was conducted in OptiMEM (Invitrogen) for $48 \mathrm{~h}$ at $37^{\circ} \mathrm{C}$ and $2 \mathrm{ml}$ DMEM was then added to the 
cell culture. The transfection efficiency was confirmed by western blotting.

For SPHK1 knockdown, small interfering RNA (siRNA) targeting SPHK1 and negative control scrambled siRNA were designed and synthesized by Guangzhou RiboBio Co., Ltd. (SPHK1-siRNA, forward, 5'-GGGCAAGGCCUUGCAGCU Cd(TT)-3' and reverse, 5'-GAGCUGCAAGGCCUUGCC Cd(TT)-3'; and scrambled siRNA, forward, 5'-UUCUCCGAA CGUGUCACGUd(TT)-3' and reverse, 5'-ACGUGACACGUU CGGAGAAd(TT)-3'). The TPC-1 cells were seeded on 6-well plates at an initial density of $5 \times 10^{4}$ cells per well and were then transfected using $50 \mathrm{nM}$ Lipofectamine ${ }^{\circledR}$ RNAiMAX (Invitrogen; Thermo Fisher Scientific, Inc.) reagent for $5 \mathrm{~min}$ at room temperature, according to the manufacturer's protocol. The concentration of siRNA duplexes was $100 \mathrm{nmol} / \mathrm{l}$. The cells were cultured in OptiMEM (Invitrogen) for $24 \mathrm{~h}$ at $37^{\circ} \mathrm{C}$ and $2 \mathrm{ml} \mathrm{DMEM}$ was then added to the cell culture. The transfection efficiency was confirmed with western blotting.

Western blotting. To detect protein expression, TPC-1 cells that had been transfected for $24 \mathrm{~h}$ were washed in PBS and lysed with radioimmunoprecipitation assay buffer $(50 \mathrm{mM}$ TrisHCl pH 7.4; 150 mM NaCl; 2 mM EDTA; 1\% NP-40; $0.1 \%$ SDS). The protein concentrations were determined using a bicinchoninic acid protein quantitation assay kit (Beyotime Institute of Biotechnology). Protein $(20 \mu \mathrm{g})$ were separated by $10 \%$ SDS-PAGE and transferred onto a PVDF nylon membrane (Merck KGaA) at $4^{\circ} \mathrm{C}$. Membranes were blocked for $30 \mathrm{~min}$ in $5 \%$ non-fat powdered milk at room temperature. After washing with TBS/Tween-20 (TBST) three times for $5 \mathrm{~min}$ each, the membranes were incubated with the anti-SPHK1 primary antibody $(1: 100$; cat. no. ab71700; Abcam) for $8 \mathrm{~h}$ at $4^{\circ} \mathrm{C}$. The membranes were then washed three times with TBST for $10 \mathrm{~min}$ each to remove free primary antibody. Mouse anti-GAPDH antibody (1:1,000 dilution; cat. no. ab8245; Abcam) was used as the loading control. After further washing, the membranes were incubated in a horseradish peroxidase-conjugated anti-rabbit IgG secondary antibody (1:1,000; cat. no. sc2004; Santa Cruz Biotechnology, Inc.) solution for $1 \mathrm{~h}$ at room temperature. All blots were visualized using an enhanced chemiluminescence solution (Merck KGaA) and analyzed using Quantity One Protein Analysis software version 4.6.7 (Bio-Rad Laboratories, Inc.).

Proliferation and invasion assay. Cell proliferation was assessed using a Cell Counting Kit-8 (CCK-8; MedChemExpress) according to the manufacturer's protocol. Briefly, the TPC-1 cells were seeded into 96-well plates at a density of $1 \times 10^{3}$ cells per well (200 $\mu \mathrm{l}$ per well). Transfected TPC-1 cells were cultured for $24 \mathrm{~h}$ and used for proliferation assay. In order to detect the relative cell proliferation rate, CCK-8 reagent was added to the medium and incubated for $3 \mathrm{~h}$ at $37^{\circ} \mathrm{C}$ in $5 \% \mathrm{CO}_{2}$. Then, a microplate reader was utilized to measure the absorbance at $450 \mathrm{~nm}$. Each experiment was performed at least three times.

Transwell inserts were used to assess the cell invasion ability. The extent of cell invasion was assessed using a BD BioCoat $^{\mathrm{TM}}$ Matrigel $^{\mathrm{TM}}$ invasion chamber with $8-\mu \mathrm{m}$ pores (BD Biosciences), according to the manufacturer's protocol.
In brief, for the invasion assay, $3 \times 10^{4}$ transfected cells in $200 \mu 1$ serum-free DMEM were seeded in the upper chambers of Matrigel-coated Transwell plates. Complete DMEM containing $10 \%$ FBS was added to the lower chambers. The Transwell chambers were then incubated at $37^{\circ} \mathrm{C}$ for $48 \mathrm{~h}$. The upper chamber was washed with PBS and cells were fixed with pure methanol for $15 \mathrm{~min}$ at room temperature. Subsequently, the cells were stained with $0.1 \%$ crystal violet for $30 \mathrm{~min}$ at room temperature. The stained cells were viewed under a light microscope (magnification, x40), images were captured and the cell number was counted in five random sights.

Colony-formation assay. Cells in the logarithmic growth phase $\left(1 \times 10^{3}\right.$ cells/well) were plated into 6 -well plates. Transfected TPC-1 cells were culture for $24 \mathrm{~h}$ before colony-formation assay. Following incubation in DMEM with $10 \% \mathrm{FBS}$ at $37^{\circ} \mathrm{C}$ for 2 weeks, the cells were washed with PBS and fixed with pure methanol for $30 \mathrm{~min}$ at room temperature, and colonies were stained with $0.1 \%$ crystal violet at room temperature for 10 min. Images were captured with an Olympus BX42 light microscope (magnification, $x 40)$. The number of clones $(\geq 50$ cells were considered to be a colony) was recorded using Image $\mathbf{J}$ software version 1.4 (National Institutes of Health).

Statistical analysis. Statistical analysis was performed using the SPSS statistical software package (version 20.0; IBM Corp.). The SPHK1 protein relative expression levels, PTC cell proliferation rate, invasion cell numbers and colony numbers were analyzed using a one-way ANOVA followed by a Fisher's least significant difference post-hoc test. A $\chi^{2}$ test was performed to analyze the association between SPHK1 expression and the clinicopathological characteristics of patients. Univariate and multivariate regression analysis was performed using the Cox proportional hazards regression model to determine the effects of potential risk factors on survival. Survival analysis was carried out using the Kaplan-Meier method and tested with the log-rank test. $\mathrm{P}<0.05$ was considered to indicate a statistically significant difference.

\section{Results}

Patient characteristics. The present study included samples from 92 patients with PTC. A total of 66 patients $(71.7 \%$ ) were female, and the mean age of the cohort was 49.2 years (range, 19-77 years). The characteristics of the patients are presented in Table I. A total of 53 patients (57.6\%) had tumors $\leq 2 \mathrm{~cm}$ in diameter, and the mean tumor size was $1.9 \mathrm{~cm}$ (range, $0.1-4.4 \mathrm{~cm}$ ). More patients presented without TCI than with it (69.6\% vs. $30.4 \%)$ and nearly half presented with ETE (48.9\%). Regional LN metastasis was reported as absent in the majority of patients $(73.9 \%$; 68/92). A total of 65 individuals were diagnosed with stage I-II $(70.7 \%)$, and $27(29.3 \%)$ with more advanced stages of the disease. The proportions of patients positive for distant metastasis, vascular invasion and recurrence were 13.0, 28.3 and $14.1 \%$, respectively.

Association between SPHK1 expression and clinicopathological characteristics. The present study aimed to determine the expression patterns of this protein in PTC tumors. First, 
Table I. Characteristics of the patients with papillary thyroid carcinoma and associations with high $(\mathrm{n}=35)$ and low $(\mathrm{n}=57)$ SPHK1 expression level.

\begin{tabular}{|c|c|c|c|c|}
\hline \multirow[b]{2}{*}{ Variable } & \multirow[b]{2}{*}{ Cases, n (\%) } & \multicolumn{2}{|c|}{ SPHK1 expression } & \multirow[b]{2}{*}{ P-value } \\
\hline & & Low, n (\%) & High, n (\%) & \\
\hline Age, years & & & & 0.262 \\
\hline$\leq 46$ & $41(44.6)$ & $28(49.1)$ & $13(37.1)$ & \\
\hline$>46$ & $51(55.4)$ & $29(50.9)$ & $22(62.9)$ & \\
\hline Sex & & & & 0.138 \\
\hline Male & $26(28.3)$ & $13(22.8)$ & $13(37.1)$ & \\
\hline Female & $66(71.7)$ & $44(77.2)$ & $22(62.9)$ & \\
\hline Tumor size, cm & & & & 0.001 \\
\hline$\leq 2$ & $53(57.6)$ & $47(82.5)$ & $6(17.1)$ & \\
\hline$>2$ & $38(41.3)$ & $10(17.5)$ & $29(82.9)$ & \\
\hline TCI & & & & 0.118 \\
\hline Negative & $64(69.6)$ & $43(75.4)$ & $21(60.0)$ & \\
\hline Positive & $28(30.4)$ & $14(24.6)$ & $14(40.0)$ & \\
\hline ETE & & & & 0.096 \\
\hline Negative & $47(51.1)$ & $33(57.9)$ & $14(40.0)$ & \\
\hline Positive & $45(48.9)$ & $24(42.1)$ & $21(60.0)$ & \\
\hline LN metastasis & & & & 0.004 \\
\hline Negative & $68(73.9)$ & $48(84.2)$ & $20(57.1)$ & \\
\hline Positive & $24(26.1)$ & $9(15.8)$ & $15(42.9)$ & \\
\hline Distant metastasis & & & & 0.005 \\
\hline Negative & $80(87.0)$ & $54(94.7)$ & $26(74.3)$ & \\
\hline Positive & $12(13.0)$ & $3(5.3)$ & $9(25.7)$ & \\
\hline TNM stage & & & & 0.001 \\
\hline I-II & $65(70.7)$ & $48(84.2)$ & $17(48.6)$ & \\
\hline III-IV & $27(29.3)$ & $9(15.8)$ & $18(51.4)$ & \\
\hline Vascular invasion & & & & 0.001 \\
\hline Negative & $66(71.7)$ & $49(86.0)$ & $17(48.6)$ & \\
\hline Positive & $26(28.3)$ & $8(14.0)$ & $18(51.4)$ & \\
\hline Recurrence & & & & 0.012 \\
\hline None & $79(85.9)$ & $53(93.0)$ & $26(74.3)$ & \\
\hline Local and distant & $13(14.1)$ & $4(7.0)$ & $9(25.7)$ & \\
\hline
\end{tabular}

SPHK1, sphingosine kinase 1; TCI, thyroid carcinoma invasion; ETE, extrathyroidal extension; LN, lymph node; TNM, Tumor-Node-Metastasis.

the protein expression levels of SPHK1 in PTC tissues were assessed using IHC on tissue microarrays that comprised of a total of 92 clinical samples. Representative IHC images exhibiting weak or strong staining for SPHK1 are presented in Fig. 1. SPHK1 protein was predominantly detected in the cytoplasm of tumor cells.

According to the percentage of positively stained cells and the staining intensity of the PTC sections, the samples were classified into low- and high-expression groups (Table I). The $\chi^{2}$ test was used to analyze the association between the characteristics of the patients with PTC and SPHK1 expression. Increased SPHK1 expression in PTC tissue was revealed to be significantly associated with tumor size, LN metastasis, TNM stage, distant metastasis, vascular invasion and recurrence (all $\mathrm{P}<0.05$ ). However, no association was observed between
SPHK1 expression and age, sex, TCI or ETE occurrence (all $\mathrm{P}>0.05$ ).

SPHK1 expression is associated with the prognosis of patients with PTC. Since high expression levels of SPHK1 were associated with larger tumor size and advanced TNM stage, it was hypothesized that this may play an important role in predicting the clinical outcome of patients with PTC. Therefore, the association between disease-free survival (DFS) time of patients and SPHK1 expression was analyzed using Kaplan-Meier analysis and the log-rank test. The results revealed that the patients that exhibited high SPHK1 expression levels had significantly shorter DFS times than those with low expression (mean DFS time, 80.3 \pm 6.9 months vs. 103.9 \pm 3.7 ; $\mathrm{P}=0.003$; Fig. $2 \mathrm{H}$ ). The prognostic value of the 

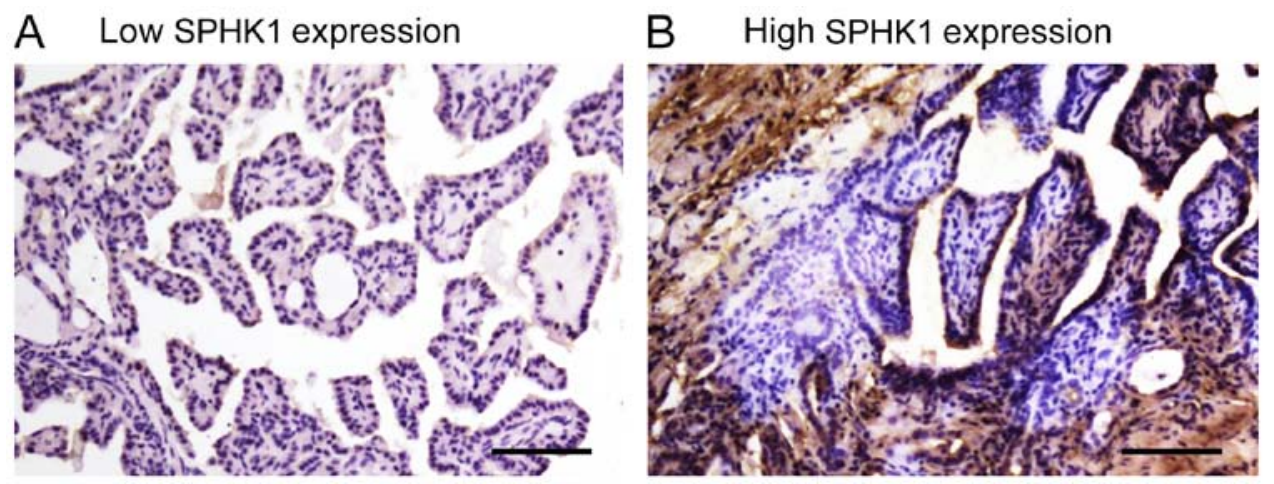

Figure 1. Representative images of human PTC tissue samples stained for SPHK1 by immunohystochemistry. (A) Low and (B) high expression of SPHK1 protein in papillary thyroid carcinoma cells, particularly in the cytoplasm. Magnification, x100. Scale bar, $100 \mu \mathrm{m}$. SPHK1, sphingosine kinase 1.

A

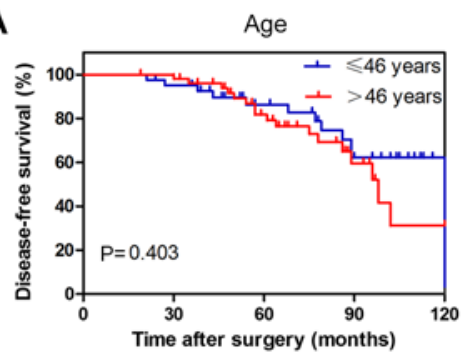

D

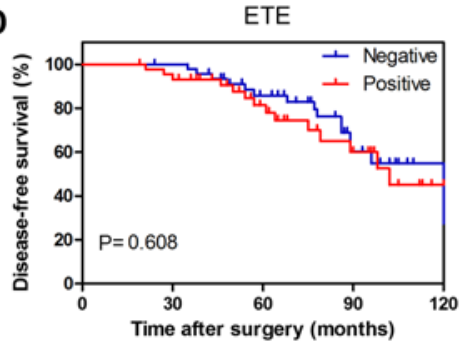

G

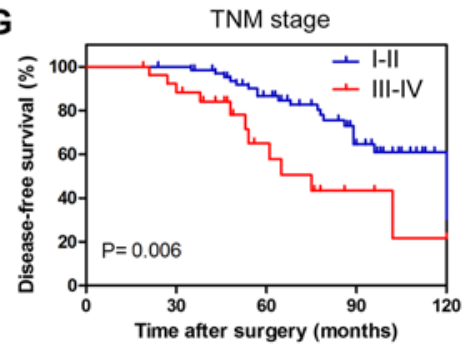

$\mathbf{J}$



B

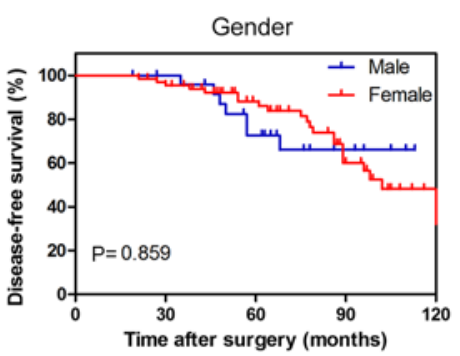

E

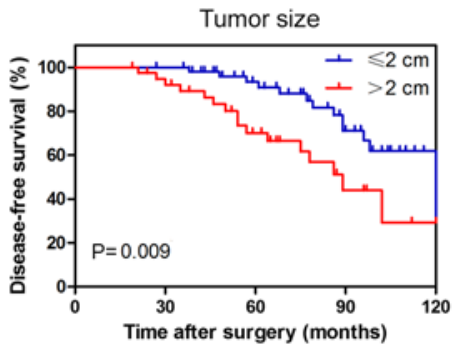

H

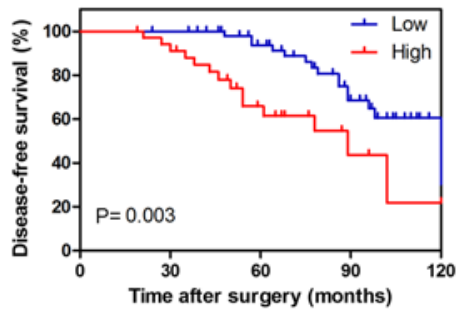

$\mathrm{K}$

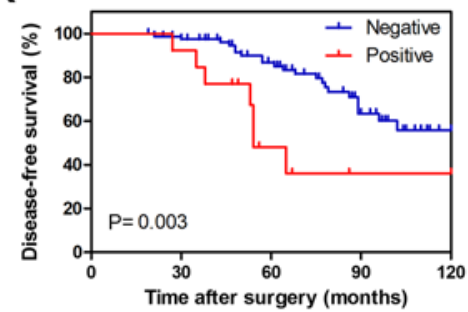

C

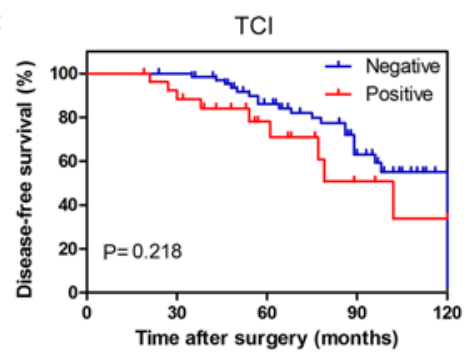

$F$
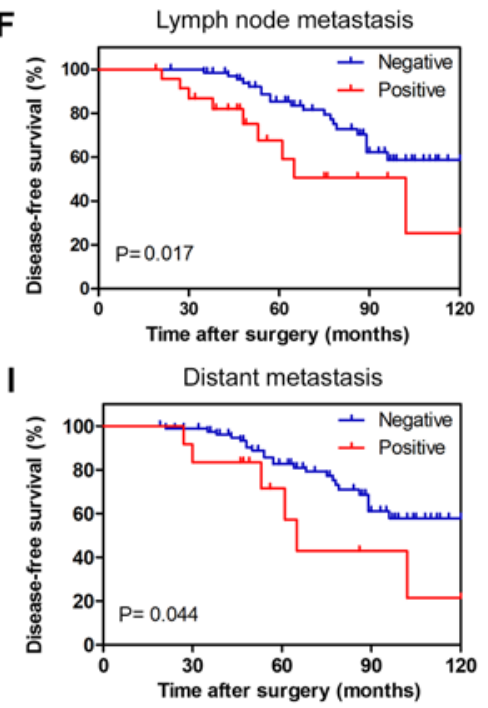

Figure 2. Kaplan-Meier curves for disease-free survival in patients with papillary thyroid carcinoma. (A) Age; (B) gender; (C) TCI; (D) ETE; (E) tumor size; (F) lymph node metastasis; (G) TNM state; (H) SPHK1 expression; (I) distant metastasis; (J) vascular invasion; (K) recurrence. Statistical significance was tested using the log-rank test. TCI, thyroid capsular invasion; ETE, extrathyroidal extension; TNM, Tumor-Node-Metastasis; SPHK1, sphingosine kinase 1.

other clinical characteristics were also analyzed. A tumor size of $\geq 2 \mathrm{~cm}$ ( $\mathrm{P}=0.009$; Fig. $2 \mathrm{E}), \mathrm{LN}$ metastasis $(\mathrm{P}=0.017$; Fig. $2 \mathrm{~F})$, advanced TNM stage ( $\mathrm{P}=0.006$; Fig. $2 \mathrm{G})$, distant metastasis
( $\mathrm{P}=0.044$; Fig. 2I), vascular invasion ( $\mathrm{P}=0.013$; Fig. $2 \mathrm{~J})$ and recurrence $(\mathrm{P}=0.003$; Fig. $2 \mathrm{~K})$ were all revealed to be unfavorable prognostic factors. However, no significant association 
Table II. Univariate and multivariate Cox regression analyses of disease-free survival in patients with papillary thyroid carcinoma.

\begin{tabular}{|c|c|c|c|c|c|c|}
\hline \multirow[b]{2}{*}{ Characteristic } & \multicolumn{3}{|c|}{ Univariate analysis } & \multicolumn{3}{|c|}{ Multivariate analysis } \\
\hline & HR & $95 \% \mathrm{CI}$ & P-value & HR & $95 \% \mathrm{CI}$ & P-value \\
\hline Age ( $\leq 46$ years vs. $>46$ years) & 0.868 & $0.487-1.302$ & 0.441 & - & - & - \\
\hline Sex (male vs. female) & 0.770 & $0.369-1.227$ & 0.335 & - & - & - \\
\hline TCI (positive vs. negative) & 1.562 & $0.937-2.210$ & 0.148 & - & - & - \\
\hline ETE (positive vs. negative) & 1.173 & $0.775-1.650$ & 0.292 & - & - & - \\
\hline LN metastasis (positive vs. negative) & 1.631 & $0.880-2.936$ & 0.351 & - & - & - \\
\hline Tumor size $(>2 \mathrm{~cm}$ vs. $\leq 2 \mathrm{~cm})$ & 4.148 & $2.134-6.279$ & 0.002 & 2.568 & $1.224-5.387$ & 0.013 \\
\hline TNM stage (I-II vs. III-IV) & 3.539 & $1.763-5.195$ & 0.003 & 2.493 & $1.382-4.330$ & 0.010 \\
\hline Distant metastasis (positive vs. negative) & 2.448 & $1.639-3.760$ & 0.013 & 1.636 & $1.275-2.994$ & 0.037 \\
\hline Vascular invasion (positive vs. negative) & 2.918 & $1.720-4.889$ & 0.001 & 2.330 & $1.378-4.195$ & 0.008 \\
\hline Recurrence (local and distant vs. none) & 2.483 & $1.704-3.955$ & 0.005 & 1.878 & $1.135-3.014$ & 0.012 \\
\hline SPHK1 (high vs. low) & 4.980 & $2.156-9.562$ & 0.001 & 3.649 & $1.584-7.371$ & 0.004 \\
\hline
\end{tabular}

HR, hazard ratio; CI, confidence interval; TCI, thyroid carcinoma invasion; ETE, extrathyroidal extension; LN, lymph node; TNM, Tumor-Node-Metastasis; SPHK1, sphingosine kinase 1.

was observed between prognosis and age, sex, TCI or ETE status (all P>0.05; Fig. 2A-D).

Subsequently, the independent hazard effect of each factor was identified using a univariate and multivariate Cox regression analysis model (Table II). Univariate Cox regression analysis indicated that tumor size, TNM stage, distant metastasis, vascular invasion, recurrence state and SPHK1 expression were significantly associated with disease-free survival time of patients with PTC $(\mathrm{P}<0.05$; Table II). Furthermore, the multivariate Cox regression analysis demonstrated that tumor size, TNM stage, distant metastasis, vascular invasion, recurrence state and SPHK1 expression were all independent prognostic factors for these patients (all $\mathrm{P}<0.05$; Table II).

SPHK1 directly promotes PTC cell proliferation and invasion. In order to investigate the role of SPHK1 in PTC cell proliferation and invasion, SPHK1 was overexpressed and downregulated in TPC-1 cells using the pcDNA3.1-SPHK1 plasmid and SPHK1-siRNA transfection. As presented in Fig. 3A-C, the protein level of SPHK1 was significantly higher in TPC-1 cells transfected with the overexpression plasmid compared with the corresponding empty vector-transfected TPC-1 cells (control), and significantly lower in the cells transfected with the siRNA compared with those transfected with the scrambled siRNA control. A CCK-8 assay was performed to test cell proliferation, which demonstrated a significantly enhanced proliferation pattern in SPHK1 overexpressing cells (Fig. 3D). In contrast, TPC-1 cells transfected with SPHK1 siRNA exhibited a strong decrease in cell viability (Fig. 3E). In addition, cell invasion was evaluated using the Transwell Matrigel invasion assay in vitro. Overexpression of SPHK1 significantly increased the invasiveness of TPC-1 cells by $\sim 2.9$ times more than the control group. Cells with SPHK1 inhibition exhibited a 53\% decrease in invasion capacity (Fig. 3F-H). Finally, colony-formation assays were also used to investigate the role of SPHK1 in TPC-1 cell survival. Fig. 3I-K demonstrate that the ability of TPC-1 cells to form colonies was significantly altered in cells with overexpressed or silenced SPHK1. Colony-formation number increased significantly in TPC-1 cells overexpressing SPHK1 compared with the empty vector control group, and decreased significantly on the knockdown of SPHK1.

\section{Discussion}

Thyroid cancer is one of the most common endocrine tumors diagnosed worldwide, with an estimated annual incidence of 12.2 cases per 100,000 individuals in the USA (22). PTC is the most common pathological type of thyroid carcinoma, and is generally characterized by an improved prognosis compared with other subtypes of thyroid carcinoma; however, the recurrence rate of PTC following the initial surgical treatment has been reported to vary by $8-23 \%(23,24)$. Therefore, investigating the underlying molecular mechanisms and various clinical factors associated with recurrence is essential in order to develop more effective diagnosis and treatment strategies for PTC. Cumulative data have revealed that SPHK1 is involved in cell proliferation, cell survival and tumorigenesis, and that it acts as a predictor of unfavorable prognosis in breast and colorectal cancers $(16,25)$. However, little is known about its role in the pathophysiology and clinical practice of PTC. In the present study, SPHK1 expression levels in human PTC tissue were investigated, in addition to its association with clinico- and histopathological features. The role of SPHK1 in the proliferation and invasion of PTC cells was also identified.

SPHK1 has previously been demonstrated to be upregulated in prostate and kidney cancers $(12,26)$. Consistent with these previous reports, the data from the present study demonstrated that SPHK1 was upregulated in $38 \%$ of the participating patients with PTC, according to tissue microarrays, and that it was significantly associated with tumor size, LN metastasis and TNM stage. Furthermore, multivariate analyses of DFS revealed that high SPHK1 expression levels, a large tumor size and an advanced TNM stage were independent predictive risk factors for DFS, indicating a higher 

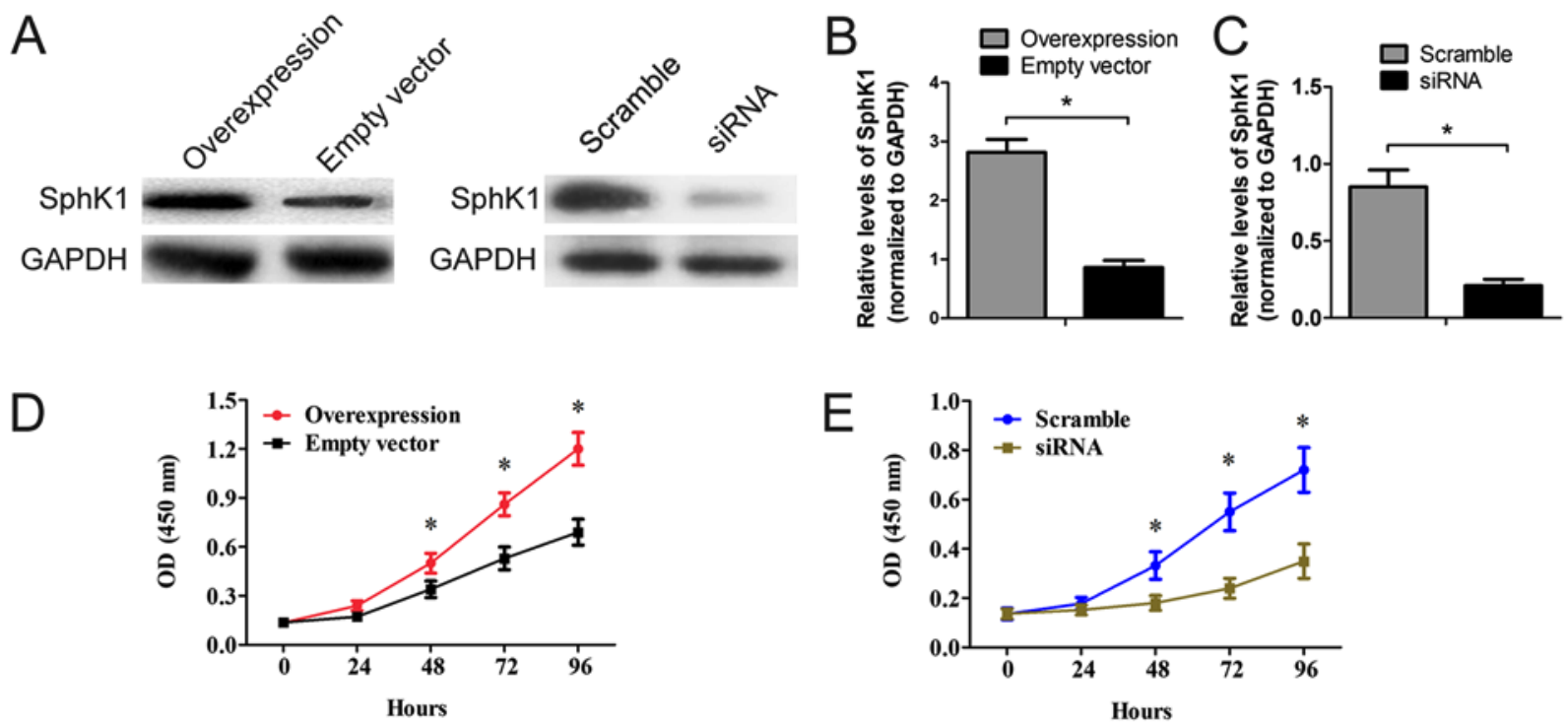
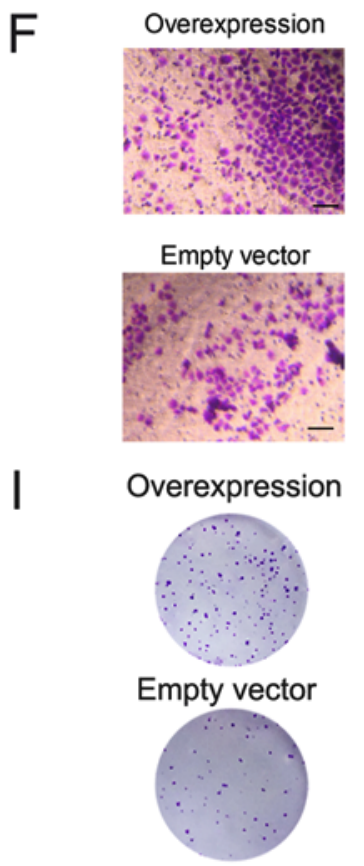



SiRNA
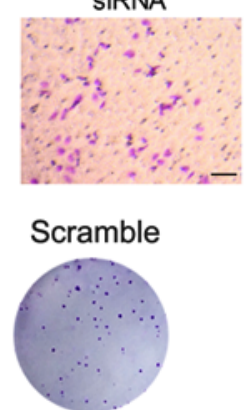

SiRNA



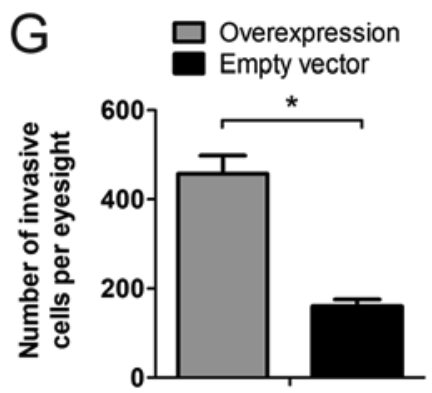

$J$

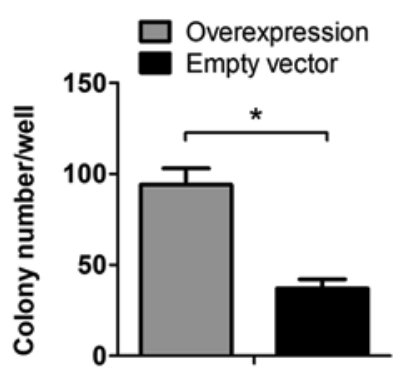

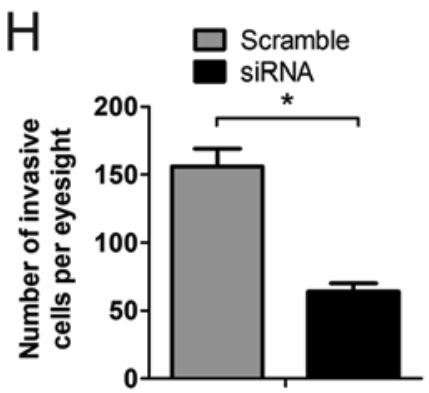

$\mathrm{K}$



Figure 3. SPHK1 promotes PTC cell proliferation and invasion. (A) The pcDNA3.1-SPHK1 overexpression plasmid and SPHK1-siRNA were transfected into TPC-1 cells, and a western blot assay was performed $24 \mathrm{~h}$ after transfection. Quantification of the relative levels of SPHK1 protein detected in the western blot assay of the cells transfected with (B) overexpression plasmin and (C) siRNA. A Cell Counting Kit-8 assay was performed to evaluate the effect of SPHK1 on the proliferation of TPC-1 cells transfected with (D) overexpression plasmin and (E) siRNA at the indicated time points. (F) Representative images of the Matrigel invasion assay. Quantification of the Matrigel invasion assay results from TPC -1 cells transfected with. Magnification, $\mathrm{x} 40$, Scale bars $=50 \mu \mathrm{m}$. $(\mathrm{G})$ the pcDNA3.1-SPHK1 plasmid or (H) SPHK1-siRNA. (I-K) (I) Representative images of the colony-formation assay, and quantification of colonies TPC-1 cells transfected with (J) the pcDNA3.1-SPHK1 plasmid or (K) SPHK1-siRNA. Magnification, x40. *P<0.05. SPHK1, sphingosine kinase 1; PTC, papillary thyroid carcinoma; siRNA, small interfering RNA.

disease recurrence risk. These results suggest that SPHK1 plays tumor-promoting roles in PTC. A previous study demonstrated that SPHK1 is capable of promoting cell invasion and proliferation in human hepatocellular carcinoma (27). Other in vivo studies have also confirmed that SPHK1 is involved in increasing sacral chordoma and esophageal carcinoma rapid cell growth in addition to spontaneous metastasis $(28,29)$. Consistent with these results, the present study demonstrated that heterogenous overexpression of SPHK1 could enhance cell proliferation and invasion processes, while SPHK1 silencing impaired cell viability and invasion. These results provide new direction for drug discovery and tumor clinical therapy.

SPHK1 can be activated by a variety of growth factors, cytokines and mitogens, such as platelet-derived growth factor (PDGF), tumor necrosis factor- $\alpha$, and vascular endothelial growth factor (VEGF) $(30,31)$. Pitson et al (32) reported that the phosphorylation of SPHK1 and its translocation from the cytoplasm to the cell membrane may be the key process responsible for inducing the malignant phenotype of cells. This process not only promotes the proliferation of malignant cells, but also protects the apoptotic pathway from being 
destroyed, thereby producing carcinogenic effects. Altering the subcellular location of SPHK1 exerts marked effects on cell function, with cell membrane-translocated SPHK1 exhibiting a potent inhibitory effect on the $G_{1}-S$ phase transition in 3T3-L1 fibroblasts, suggesting that the localization of SPHK1 in cells may have an effect on tumor cell apoptosis. Ogretmen (33) determined the metabolism of ceramide for S1P biosynthesis, which is mediated by SPHK1 and -2 , and its role in influencing cancer cell growth, drug resistance and tumor metastasis through S1PR-dependent or receptor-independent signaling. In the present study, it was observed that overexpression of SPHK1 promotes the malignant biological behavior of TPC-1 cells. Recently, increasing evidence has indicated that SPHK1 acts as an enzyme involved in carcinogenesis $(34,35)$. The potential underlying molecular mechanisms of SPHK1 in the pathogenesis of PTC may include its enzymatic activity of upregulating S1P. S1P, a bioactive lipid, potentially contributes to tumorigenesis. S1P can bind to a family of $\mathrm{G}$ protein-coupled receptors (S1PRs) to induce cellular responses such as the survival, proliferation, apoptosis and migration of cancer cells (35).

SPHK1 overexpression functions as a prognostic marker for judging the survival time of patients with different types of cancer (36). However, the roles of SPHK1 have not been extensively investigated, particularly in the long-term DFS time of patients with PTC. To this effect, the present study investigated the associations between SPHK1 expression levels and the clinical characteristics of patients with PTC, and the results revealed a significant association between SPHK1 expression and tumor size, clinical stage and LN metastasis. Notably, there was a significant association between a shorter DFS time of patients with PTC and high SPHK1 expression, suggesting that SPHK1 may be a useful prognostic marker for patients with PTC. The prognostic role of SPHK1 has also been revealed in numerous types of cancer, including astrocytoma, breast cancer and gastric cancer, suggesting that patients with low levels of SPHK1 expression have longer overall survival time, while those with higher SPHK1 expression levels survived for shorter durations $(16,37,38)$. In addition, Rosa et al (39) reported that SPHK1 overexpression contributes to cetuximab resistance in human colorectal cancer models. Furthermore, multivariate analysis of the results from the present study confirmed that SPHK1 expression was an independent factor for predicting DFS of patients with PTC. Consistent with the observations in the present study, additional studies demonstrated that SPHK1 upregulation was an independent prognostic factor for nasopharyngeal carcinoma, salivary gland carcinoma, breast cancer and hepatocellular carcinoma $(16,17,40)$. However, the present study had certain limitations, including a lack of an intensive investigation of the molecular mechanisms underlying the associations between SPHK1 expression and tumor occurrence and metastasis in patients with PTC. Another limitation of the present study is the lack of investigation of S1P, which is released by SPHK1 activity and binds to S1P receptors, which has been demonstrated to participate in the epithelial-to-mesenchymal transition of a number of different types of tumor (41). Future studies must elucidate the potential molecular mechanisms underlying SPHK1 in PTC, using a much larger sample size and longer follow-up times.
In summary, the present study demonstrated that increased SPHK1 expression levels were significantly associated with the progression and poor prognosis of patients with PTC, indicating that SPHK1 may represent a novel and valuable predictor for the prognosis of patients with PTC. In addition, the present study demonstrated that overexpression of SPHK1 with the pcDNA3.1-SPHK1 plasmid results in the enhanced malignant behavior of PTC cells in vitro. Further studies on the possible molecular mechanisms of SPHK1 participation in PTC tumor progression may ultimately lead to the development of a new potent anti-PTC strategy.

\section{Acknowledgements}

Not applicable.

\section{Funding}

The present study was supported by the Hebei Cangzhou Science and Technology Plan Project of China (grant no. 131302136).

\section{Availability of data and materials}

The datasets used and/or analyzed during the current study are available from the corresponding author on reasonable request.

\section{Authors' contributions}

JL designed the study. JL, BZ, YB, YL, BZ and JJ performed the experiments and analyzed the data. JL and BZ wrote the manuscript. BZ and JJ helped to revise the manuscript. All authors read and approved the final version of the manuscript and agree to be accountable for all aspects of the research in ensuring that the accuracy or integrity of any part of the work are appropriately investigated and resolved.

\section{Ethics approval and consent to participate}

The protocol for the present study was approved by the Medical Institutional and Clinical Research Ethics Committee of Cangzhou Central Hospital (Cangzhou, China). All patients included in the present study previously provided written informed consent.

\section{Patient consent for publication}

Not applicable.

\section{Competing interests}

The authors declare that they have no conflicts of interest.

\section{References}

1. Al-Brahim N and Asa SL: Papillary thyroid carcinoma: An overview. Arch Pathol Lab Med 130: 1057-1062, 2006.

2. Nikiforova MN and Nikiforov YE: Molecular genetics of thyroid cancer: Implications for diagnosis, treatment and prognosis. Expert Rev Mol Diagn 8: 83-95, 2008. 
3. Siegel RL, Miller KD and Jemal A: Cancer statistics, 2015. CA Cancer J Clin 65: 5-29, 2015.

4. Enewold L, Zhu K, Ron E, Marrogi AJ, Stojadinovic A, Peoples GE and Devesa SS: Rising thyroid cancer incidence in the United States by demographic and tumor characteristics, 1980-2005. Cancer Epidemiol Biomarkers Prev 18: 784-791, 2009.

5. Wang LY and Ganly I: Nodal metastases in thyroid cancer: Prognostic implications and management. Future Oncol 12 : 981-994, 2016.

6. Asimakopoulos P, Nixon IJ and Shaha AR: Differentiated and medullary thyroid cancer: Surgical management of cervical lymph nodes. Clin Oncol (R Coll Radiol) 29: 283-289, 2017.

7. Grant CS: Recurrence of papillary thyroid cancer after optimized surgery. Gland Surg 4: 52-62, 2015.

8. Giusca SE, Amalinei C, Lozneanu L, Ciobanu Apostol D, Andriescu EC, Scripcariu A, Balan R, Avadanei ER and Căruntu ID: Heterogeneous periostin expression in different histological variants of papillary thyroid carcinoma. Biomed Res Int 2017: 8701386, 2017

9. Spiegel S and Milstien S: Functions of the multifaceted family of sphingosine kinases and some close relatives. J Biol Chem 282: 2125-2129, 2007.

10. Spiegel S and Milstien S: Sphingosine-1-phosphate: An enigmatic signalling lipid. Nat Rev Mol Cell Biol 4: 397-407, 2003

11. Green JA, Suzuki K, Cho B, Willison LD, Palmer D, Allen CD, Schmidt TH, Xu Y, Proia RL, Coughlin SR and Cyster JG: The sphingosine 1-phosphate receptor $\mathrm{S}_{1} \mathrm{P}_{2}$ maintains the homeostasis of germinal center $\mathrm{B}$ cells and promotes niche confinement. Nat Immunol 12: 672-680, 2011.

12. Malavaud B, Pchejetski D, Mazerolles C, de Paiva GR, Calvet C, Doumerc N, Pitson S, Rischmann P and Cuvillier O: Sphingosine kinase-1 activity and expression in human prostate cancer resection specimens. Eur J Cancer 46: 3417-3424, 2010.

13. Bayerl MG, Bruggeman RD, Conroy EJ, Hengst JA, King TS, Jimenez M, Claxton DF and Yun JK: Sphingosine kinase 1 protein and mRNA are overexpressed in non-Hodgkin lymphomas and are attractive targets for novel pharmacological interventions. Leuk Lymphoma 49: 948-954, 2008.

14. French KJ, Schrecengost RS, Lee BD, Zhuang Y, Smith SN, Eberly JL, Yun JK and Smith CD: Discovery and evaluation of inhibitors of human sphingosine kinase. Cancer Res 63 : 5962-5969, 2003.

15. Van Brocklyn JR, Jackson CA, Pearl DK, Kotur MS, Snyder PJ and Prior TW: Sphingosine kinase-1 expression correlates with poor survival of patients with glioblastoma multiforme: Roles of sphingosine kinase isoforms in growth of glioblastoma cell lines. J Neuropathol Exp Neurol 64: 695-705, 2005

16. Zhu YJ, You H, Tan JX, Li F, Qiu Z, Li HZ, Huang HY, Zheng K and Ren GS: Overexpression of sphingosine kinase 1 is predictive of poor prognosis in human breast cancer. Oncol Lett 14 63-72, 2017.

17. Li W, Tian Z, Qin H, Li N, Zhou X, Li J, Ni B and Ruan Z: High expression of sphingosine kinase 1 is associated with poor prognosis in nasopharyngeal carcinoma. Biochem Biophys Res Commun 460: 341-347, 2015

18. Tran B, Roshan D, Abraham E, Wang L, Garibotto N, Wykes J, Campbell P and Ebrahimi A: An analysis of the American Joint Committee on Cancer 8th edition T staging system for papillary thyroid carcinoma. J Clin Endocrinol Metab 103: 2199-2206, 2018.

19. Haugen BR, Alexander EK, Bible KC, Doherty GM, Mandel SJ, Nikiforov YE, Pacini F, Randolph GW, Schlumberger M, Sawka AM, et al: 2015 American thyroid association management guidelines for adult patients with thyroid nodules and differentiated thyroid cancer: The American thyroid association guidelines task force on thyroid nodules and differentiated thyroid cancer. Thyroid 26: 1-133, 2016.

20. Chen L, Zhang W, Yan W, Han L, Zhang K, Shi Z, Zhang J, Wang Y, Li Y, Yu S, et al: The putative tumor suppressor miR-524-5p directly targets Jagged-1 and Hes-1 in glioma. Carcinogenesis 33: 2276-2282, 2012.

21. Wang HG, Cao B, Zhang LX, Song N, Li H, Zhao WZ, Li YS, Ma SM and Yin DJ: KLF2 inhibits cell growth via regulating HIF-1a/Notch-1 signal pathway in human colorectal cancer HCT116 cells. Oncol Rep 38: 584-590, 2017.

22. Nguyen QT, Lee EJ, Huang MG, Park YI, Khullar A and Plodkowski RA: Diagnosis and treatment of patients with thyroid cancer. Am Health Drug Benefits 8: 30-40, 2015.
23. Popadich A, Levin O, Lee JC, Smooke-Praw S, Ro K, Fazel M, Arora A, Tolley NS, Palazzo F, Learoyd DL, et al: A multicenter cohort study of total thyroidectomy and routine central lymph node dissection for cNO papillary thyroid cancer. Surgery 150 : 1048-1057, 2011.

24. Hartl DM, Mamelle E, Borget I, Leboulleux S, Mirghani H and Schlumberger M: Influence of prophylactic neck dissection on rate of retreatment for papillary thyroid carcinoma. World J Surg 37: 1951-1958, 2013.

25. Bae GE, DO SI, Kim K, Park JH, Cho S and Kim HS: Increased sphingosine kinase 1 expression predicts distant metastasis and poor outcome in patients with colorectal cancer. Anticancer Res 39: 663-670, 2019.

26. Bouquerel P, Gstalder C, Müller D, Laurent J, Brizuela L, Sabbadini RA, Malavaud B, Pyronnet S, Martineau Y, Ader I and Cuvillier O: Essential role for SphK1/S1P signaling to regulate hypoxia-inducible factor $2 \mathrm{a}$ expression and activity in cancer. Oncogenesis 5: e209, 2016.

27. Bao M, Chen Z, Xu Y, Zhao Y, Zha R, Huang S, Liu L, Chen T, $\mathrm{Li}$ J, Tu $\mathrm{H}$ and He X: Sphingosine kinase 1 promotes tumour cell migration and invasion via the S1P/EDG1 axis in hepatocellular carcinoma. Liver Int 32: 331-338, 2012.

28. Pan J, Tao YF, Zhou Z, Cao BR, Wu SY, Zhang YL, Hu SY, Zhao WL, Wang J, Lou GL, et al: An novel role of sphingosine kinase-1 (SPHK1) in the invasion and metastasis of esophageal carcinoma. J Transl Med 9: 157, 2011.

29. Zhang K, Chen H, Wu G, Chen K and Yang H: High expression of SPHK1 in sacral chordoma and association with patients' poor prognosis. Med Oncol 31: 247, 2014

30. Xia P, Wang L, Moretti PA, Albanese N, Chai F, Pitson SM, D'Andrea RJ, Gamble JR and Vadas MA: Sphingosine kinase interacts with TRAF2 and dissects tumor necrosis factor-alpha signaling. J Biol Chem 277: 7996-8003, 2002.

31. Wattenberg BW, Pitson SM and Raben DM: The sphingosine and diacylglycerol kinase superfamily of signaling kinases: Localization as a key to signaling function. J Lipid Res 47: 1128-1139, 2006.

32. Pitson SM, Xia P, Leclercq TM, Moretti PA, Zebol JR, Lynn HE, Wattenberg BW and Vadas MA: Phosphorylation-dependent translocation of sphingosine kinase to the plasma membrane drives its oncogenic signalling. J Exp Med 201: 49-54, 2005.

33. Ogretmen B: Sphingolipid metabolism in cancer signalling and therapy. Nat Rev Cancer 18: 33-50, 2018.

34. Nema R, Vishwakarma S, Agarwal R, Panday RK and Kumar A Emerging role of sphingosine-1-phosphate signaling in head and neck squamous cell carcinoma. Onco Targets Ther 9: 3269-3280, 2016.

35. Pyne S and Pyne NJ: Sphingosine 1-phosphate signalling in mammalian cells. Biochem J 349: 385-402, 2000.

36. Safadi-Chamberlain F, Wang LP, Payne SG, Lim CU, Stratford S, Chavez JA, Fox MH, Spiegel S and Summers SA: Effect of a membrane-targeted sphingosine kinase 1 on cell proliferation and survival. Biochem J 388: 827-834, 2005.

37. Li W, Yu CP, Xia JT, Zhang L, Weng GX, Zheng HQ, Kong QL, $\mathrm{Hu}$ LJ, Zeng MS, Zeng YX, et al: Sphingosine kinase 1 is associated with gastric cancer progression and poor survival of patients. Clin Cancer Res 15: 1393-1399, 2009.

38. Li J, Guan HY, Gong LY, Song LB, Zhang N, Wu J, Yuan J, Zheng YJ, Huang ZS and Li M: Clinical significance of sphingosine kinase-1 expression in human astrocytomas progression and overall patient survival. Clin Cancer Res 14: 6996-7003, 2008.

39. Rosa R, Marciano R, Malapelle U, Formisano L, Nappi L, D'Amato C,D'Amato V,Damiano V,Marfè G, Del Vecchio S, et al: Sphingosine kinase 1 overexpression contributes to cetuximab resistance in human colorectal cancer models. Clin Cancer Res 19: 138-147, 2013.

40. Wang F and Wu Z: Sphingosine kinase 1 overexpression is associated with poor prognosis and oxaliplatin resistance in hepatocellular carcinoma. Exp Ther Med 15: 5371-5376, 2018.

41. Brizuela L, Ader I, Mazerolles C, Bocquet M, Malavaud B and Cuvillier O: First evidence of sphingosine 1-phosphate lyase protein expression and activity downregulation in human neoplasm: Implication for resistance to therapeutics in prostate cancer. Mol Cancer Ther 11: 1841-1851, 2012.

This work is licensed under a Creative Commons Attribution-NonCommercial-NoDerivatives 4.0 International (CC BY-NC-ND 4.0) License. 